\title{
ON ANALYTIC CHARACTERISTIC FUNCTIONS
}

\author{
EUGENE LUKaCS AND OTtO SZaś
}

1. Introduction. In this paper we discuss certain properties of characteristic functions. Theorem 1 gives a sufficient condition on the characteristic function of a distribution in order that the moments of the distribution should exist. The existence of the moments is usually proven under the assumption that the characteristic function is differentiable [4]. The condition of Theorem 1 is somewhat more general and the proof shorter and more elementary. The remaining theorems deal with analytic characteristic functions, and again some known results are proved in a simple manner. Some applications are discussed; in particular, it is shown that an analytic characteristic function of an infinitely divisible law can have no zeros inside its strip of convergence. This property is used to construct an example where an infinitely divisible law (the Laplace distribution) is factored into two noninfinitely divisible factors.

2. An existence theorem. Let $F(x)$ be a probability distribution, that is, a never-decreasing, right-continuous function such that $F(-\infty)=0$ and $F(+\infty)=1$. The Fourier transform of $F(x)$, that is, the function

$$
\phi(t)=\int_{-\infty}^{+\infty} e^{i t x} d F(x)
$$

is called the characteristic function of the distribution $F(x)$. The characteristic function exists for real values of $t$ for any distribution, but the integral (1.1) does not always exist for complex $t$. This paper deals mostly with characteristic functions which are analytic in a neighborhood of the origin.

For an arbitrary function $f(y)$, we denote in the following the first difference by

$$
\Delta_{1} f(y ; t)=\Delta f(y ; t)=f(y+t)-f(y-t),
$$

and define the higher differences by

$$
\Delta_{k+1} f(y ; t)=\Delta \Delta_{k} f(y ; t)
$$

Received June 23, 1952.

Pacific J. Math. 3 (1953), 615-625 
for $k=1,2, \ldots$. It can then easily be shown that

$$
\Delta_{n} f(y ; t)=\sum_{k=0}^{n}(-1)^{k}\left(\begin{array}{l}
n \\
k
\end{array}\right) f[y+(n-2 k) t] .
$$

In particular, for the function $f(y)=e^{i m y}$ we have

$$
\Delta_{n} f(y ; t)=e^{i m y}\left(e^{i m t}-e^{-i m t}\right)^{n}=e^{i m y}[2 i \sin m t]^{n} .
$$

We first prove two lemmas.

LEMMA 1. Let $\phi(t)$ be the characteristic function of a probability distribution $F(x)$, and let $\Delta_{2 k} \phi(0 ; t) /(2 t)^{2 k}$ be the $2 k$ th difference quotient of $\phi(t)$ at the origin. Assume that

$$
\liminf _{t \rightarrow \infty}\left|\frac{\Delta_{2 k} \phi(0 ; t)}{(2 t)^{2 k}}\right|<\infty
$$

Then the $2 k$ th moment $m_{2 k}$ of the distribution $F(x)$ exists, as do all the moments $m_{r}$ of order $r<2 k$.

LEMMA 2. Under the assumptions of Lemma 1 , the derivatives $\phi^{(r)}(t)$ exist for all $t$ and for $r=1,2, \ldots, 2 k$; and

$$
\dot{\phi}^{(r)}(t)=i^{r} \int_{-\infty}^{+\infty} x^{r} e^{i t x} d F(x)
$$

Moreover, $\left|\phi^{(2 r)}(t)\right| \leq\left|\phi^{(2 r)}(0)\right|=m_{2 r}$ for $r=1,2, \cdots, k$.

Proof. The assumption of Lemma 1 means that there is a constant $M<\infty$ such that

$$
\liminf _{t \rightarrow 0}\left|\frac{\Delta_{2 k} \phi(0 ; t)}{(2 t)^{2 k}}\right|=M
$$

From (1.1) it is seen that for

$$
\phi(y)=\int_{-\infty}^{+\infty} e^{i y x} d F(x)
$$

we have 


$$
\Delta_{2 k} \phi(y ; t)=\int_{-\infty}^{+\infty} e^{i x y}[2 i \sin x t]^{2 k} d F(x)
$$

and

$$
\left|\frac{\Delta_{2 k} \phi(0 ; t)}{(2 t)^{2 k}}\right|=\int_{-\infty}^{+\infty}\left(\frac{\sin x t}{t}\right)^{2 k} d F(x) .
$$

We see therefore from $(1.2)$ that

$$
M=\liminf _{t \rightarrow 0} \int_{-\infty}^{+\infty}\left(\frac{\sin x t}{t}\right)^{2 k} d F(x)
$$

and hence that

$$
M \geq \liminf _{t \rightarrow 0} \int_{-a}^{+a}\left(\frac{\sin x t}{t}\right)^{2 k} d F(x)=\int_{-a}^{+a} x^{2 k} d F(x)
$$

for any finite $a$. It follows then that the $2 k$ th moment

$$
m_{2 k}=\int_{-\infty}^{+\infty} x^{2 k} d F(x)
$$

exists and that $M \geq m_{2 k}$. Let next $r$ be a positive integer such that $r<k$; then $x^{2 k}>x^{2 r}$ if $|x|>1$, and

$$
m_{2 k}>\int_{|x|>1} x^{2 k} d F(x)>\int_{|x|>1} x^{2 r} d F(x)
$$

so that the moments of even order $m_{2 r}[r=1,2, \ldots,(k-1)]$ exist also. Moreover,

$$
\int_{a}^{b}\left|x^{2 r-1}\right| d F(x) \leq \frac{1}{2} \int_{a}^{b}\left(x^{2 r}+x^{2 r-2}\right) d F(x) \leq \frac{1}{2}\left[m_{2 r}+m_{2 r-2}\right]
$$

for any $a$ and $b$. This shows that the absolute moments of odd order not exceeding $2 k$, and therefore also the moments $m_{2 r-1}(r=1,2, \cdots, k)$, exist. This proves Lemma 1.

From the existence of the moments $m_{r}(r=1,2, \ldots, 2 k)$ we see immediately that $\int_{-\infty}^{+\infty} x^{r} e^{i t x} d F(x)$ exists and converges absolutely and uniformly for all real $t$ and $r \leq 2 k$. It follows then from a well-known theorem (see for instance 
[2, pp.67-68]) that all derivatives exist and are obtained by differentiating under the integral sign. This proves Lemma 2.

From Lemma 1 and 2 we obtain immediately:

THEOREM 1. Let $\phi(t)$ be the characteristic function of a distribution $F(x)$, and assume that, for an infinite sequence of even integers $\left\{2 n_{k}\right\}$,

$$
\liminf _{t \rightarrow 0}\left|\frac{\Delta_{2 n_{k}} \phi(0 ; t)}{(2 t)^{2 n_{k}}}\right|=M_{k}
$$

is finite (not necessarily bounded) for $k=1,2, \ldots$. Then all the moments $m_{r}$ of the distribution $F(x)$ exist; and $\phi(t)$ can be differentiated for all real $t$ any number of times, with

$$
\phi^{(r)}(t)=i^{r} \int_{-\infty}^{+\infty} x^{r} e^{i t x} d F(x)
$$

COROLLARY 1. If all the derivatives of the characteristic function exist at the origin, then all the moments of the distribution exist.

This corollary was proved by R. Fortet [4]; it is also stated in some text books of probability [2, 5], as well as in a paper by P. Lévy [7]. Theorem 1 is somewhat more general; the proof given here is similar to the proof indicated for the corollary by H. Cramér [2, p. 89].

3. Analytic characteristic functions. From now on we assume that the characteristic function $\phi(t)$ coincides with an analytic function in some neighborhood of the origin. Then the assumptions of the corollary are satisfied, all the moments exist, and the characteristic function has the expansion

$$
\phi(z)=\sum_{k=0}^{\infty} \frac{i^{k} m_{k}}{k !} z^{k} \quad \text { for }|z|<\rho,
$$

where $\rho>0$ is the radius of convergence of the series.

We write

$$
\phi_{0}(z)=\frac{1}{2}[\phi(z)+\phi(-z)]
$$

for the even part of $\phi(z)$, and 


$$
\phi_{1}(z)=\frac{1}{2}[\phi(z)-\phi(-z)]
$$

for the odd part of $\phi(z)$, then the two series

$$
\left\{\begin{array}{l}
\phi_{0}(z)=\sum_{k=0}^{\infty} \frac{(-1)^{k} m_{2 k}}{(2 k) !} z^{2 k}, \\
\phi_{1}(z)=\sum_{k=1}^{\infty} \frac{i^{2 k-1} m_{2 k-1}}{(2 k-1) !} z^{2 k-1}
\end{array}\right.
$$

converge also in circles about the origin. Denote the radii of convergence of these series by $\rho_{0}$ and $\rho_{1}$.

If we denote the $k$ th absolute moment of $F(x)$ by

$$
\beta_{k}=\int_{-\infty}^{+\infty}|x|^{k} d F(x)
$$

and observe that

$$
\left|x^{2 k-1}\right| \leq \frac{1}{2}\left(x^{2 k}+x^{2 k-2}\right)
$$

we see that

$$
\frac{m_{2 k-1}}{(2 k-1) !} \leq \frac{\beta_{2 k-1}}{(2 k-1) !} \leq \frac{1}{2}\left[\frac{m_{2 k}}{(2 k) !}(2 k)+\frac{m_{2 k-2}}{(2 k-2) !}\right] \text {. }
$$

This shows that

$$
\rho_{1} \geq \rho_{0} \geq \rho
$$

We see further from $(2.3)$ and $\beta_{2 k}=m_{2 k}$ that the series $\sum_{k=0}^{\infty} \beta_{k} z^{k} / k$ ! converges for $|z|<\rho_{0}$. From Lemma 2 we see, for any real $\xi$, that

$$
\left|\phi^{(2 k)}(\xi)\right| \leq m_{2 k}
$$

Hence if we denote the radius of convergence of the Taylor series of $\phi_{0}(z)$ around $\xi$ by $\rho_{0}(\xi)$, then

$$
\rho_{0}(\xi) \geq \rho_{0}(0)=\rho_{0}
$$


Similarly it follows from

$$
\left|\phi^{(2 k-1)}(\xi)\right| \leq \beta_{2 k-1}
$$

and (2.3) that

$$
\rho_{1}(\xi) \geq \rho_{1}(0)=\rho_{1} \geq \rho
$$

We have thus shown that the Taylor series of $\phi_{0}(z)$ and also that of $\phi_{1}(z)$ around $\xi$ converge in circles of radii at least equal to $\rho$. The same is therefore true for the expansion of $\phi(z)$ around $\xi$; thus we conclude that the function $\phi(z)$ is analytic at least in the strip

$$
-\rho<d(z)<+\rho
$$

The analyticity of $\phi(z)$ in a horizontal strip follows also from a result of R. P. Boas [1]. Boas showed that the Fourier-Stieltjes transform of a bounded and never-decreasing function is analytic in a horizontal strip provided that it is analytic in a neighborhood of the origin.

We show next that the representation of the characteristic function by the Fourier integral $(1.1)$ is valid in the strip $-\rho<d(z)<++\rho$.

We saw above that the series $\sum_{v=0}^{\infty}|y|^{v} \beta_{v} / v$ ! converges for $|y|<\rho$. Clearly,

$$
\sum_{v=0}^{\infty} \frac{|y|^{v} \beta_{v}}{v !} \geq \sum_{v=0}^{\infty} \frac{|y|^{v}}{v !} \int_{-A}^{+A}|x|^{v} d F(x)=\int_{-A}^{+A} e^{|y x|} d F(x)
$$

for any $A$. Therefore the integral $\int_{-\infty}^{+\infty} e^{|y x|} d F(x)$ exists, and hence the integral $\int_{-\infty}^{+\infty} e^{i z x} d F(x)$ is convergent whenever $\left|e^{i z x}\right| \leq e^{|y x|}$, where $z=\zeta+i y$. Thus for any $\zeta$ and $|y|<\rho$ the integral is convergent. This integral is an analytic function in its strip of convergence and agrees with $\phi(z)$ for real $z$; therefore it must agree with $\phi(z)$ also for complex values $z=\zeta+i y$ provided $|y|<\rho$.

We are now in a position to formulate our main result.

THEOREM 2. If a characteristic function $\phi(z)$ is analytic in a neighborhood of the origin, then it is also analytic in a horizontal strip and can be represented in this strip by a Fourier integral. Either this strip is the whole plane, or it has one or two horizontal boundary lines. The purely imaginary points on the boundary 
of the strip of convergence (if it is not the whole plane) are singular points of $\phi(z)$.

The first part of Theorem 2 was established above; we have to prove the statement concerning the singular points of $\phi(z)$.

The integral

$$
\phi(z)=\int_{-\infty}^{+\infty} e^{i z x} d F(x)
$$

converges in a strip $-\alpha<d(z)<+\beta$, where $\alpha \geq \rho$ and $\beta \geq \rho$, and is analytic inside this strip. To carry out the proof concerning the singular points of $\phi(z)$, we use the decomposition

$$
\phi(z)=\int_{0}^{\infty} e^{i z x} d F(x)+\int_{-\infty}^{0} e^{i z x} d F(x)=\mathcal{L}_{1}(z)+\mathcal{L}_{2}(z) \quad \text { (say). }
$$

Now $\mathcal{L}_{1}(z)$ and $\mathcal{L}_{2}(z)$ are Laplace integrals, convergent in the half-planes $d(z)>-\alpha$ and $d(z)<\beta$, respectively. Let $z=i w$; then $i z=-w$. If $z=\zeta+i y$, then $w=-i \zeta+y$; thus

$$
\mathcal{L}_{1}(i w)=\int_{0}^{\infty} e^{-w x} d F(x) \equiv \Phi(w)
$$

is convergent for $K(w)>-\alpha$.

It is known that the Laplace transform

$$
g(s)=\int_{0}^{\infty} e^{-s t} d G(t)
$$

of a monotonic function $G(t)$ has a singularity at the real point of its axis of convergence. For a proof the reader is referred to [9, p.58]. This theorem is similar to well-known theorems in power series and Dirichlet series. From the fact that $F(x)$ is nondecreasing we conclude therefore that $-\alpha$ is a singular point of $\Phi(w)$. Thus $-i \alpha$ is a singular point of $\phi(z)$. In the same way it is also seen that $i \beta$ is a singular point of $\phi(z)$.

Theorem 2 was stated without proof in a recent paper by D. Dugué [3], and is indicated in a footnote of an earlier paper by P. Lévy [7].

An immediate consequence of the preceding result is this:

CoROLLARY 2. A necessary condition that a function analytic in some 
neighborhood of the origin be a characteristic function is that in either half-plane the singularity nearest to the real axis be located on the imaginary axis.

4. Applications. In the following we discuss some applications of our new results.

The corollary to Theorem 2 can sometimes be used to decide whether the the quotient of two characteristic functions is again a characteristic function. We illustrate this by an example. Let

$$
\phi_{1}(t)=\left[\left(1-\frac{i t}{a}\right)\left(1-\frac{i t}{a+i b}\right)\left(1-\frac{i t}{a-i b}\right)\right]^{-1}
$$

and

$$
\phi_{2}(t)=\left[1-\frac{i t}{a}\right]^{-1},
$$

with $a^{2} \geq b^{2}>0$. It is easy to see that both these functions are characteristic functions. Their quotient

$$
\phi(t)=\frac{\phi_{1}(t)}{\phi_{2}(t)}
$$

satisfies the elementary necessary conditions for characteristic functions, namely $\phi(-t)=\overline{\phi(t)}, \phi(0)=1,|\phi(t)| \leq 1$ for real $t$. However, the condition of the corollary to Theorem 2 is violated since $\phi(t)$ has no singularity on the imaginary axis while it has a pair of conjugate complex poles $\pm b-i a$. Therefore $\phi(t)$ can not be a characteristic function.

Theorem 2 can also be used to establish the following property of analytic characteristic functions.

THEOREM 3. Let $\phi(z)$ be an analytic characteristic function. Then for any horizontal line in the strip, the function $\phi(z)$ and its derivatives $\phi^{(k)}(z)$ all attain their absolute maxima on the imaginary axis.

Proof. By Theorem 2 we have

$$
\phi(z)=\int_{-\infty}^{+\infty} e^{i x z} d F(x)
$$

in the strip of convergence. Let $z=i a+\eta$; then 


$$
\max _{-\infty<\eta<\infty}|\phi(i a+\eta)| \leq \int_{-\infty}^{+\infty} e^{-a x} d F(x)=\phi(i a)
$$

This result is due to Dugue' [3].

We also obtain:

COROLLARY 3. An analytic characteristic function has no zeros on the segment of the imaginary axis inside the strip of analyticity. The zeros and the singular points of $\phi(z)$ are located symmetrically with respect to the imaginary axis.

The first part of the corollary follows immediately from Theorem 3; we obtain the statement about the location of the zeros and singularities of $\phi(z)$ if we observe that the functional relation

$$
\phi(z)=\overline{\phi(-\bar{z})}
$$

holds not only in the strip of convergence of the Fourier integral but in the entire domain of regularity of $\phi(z)$.

An important theorem on analytic characteristic functions is due to P. Lévy [6] and D. Raikov [8]:

THEOREM OF LÉVY AND RAIKOV. Let $\phi(t)$ be an analytic characteristic function, and assume that $\phi(t)=\phi_{1}(t) \phi_{2}(t)$, where $\phi_{1}(t)$ and $\phi_{2}(t)$ are both characteristic functions. Then the factors $\phi_{1}(t)$ and $\phi_{2}(t)$ are also analytic functions, and their representations as Fourier integrals converge at least in the strip of convergence of $\phi(t)$.

This theorem was originally proven by P. Lévy [6; 7] only for entire characteristic functions; a simple proof may be found in [3].

From the foregoing theorem we easily deduce:

THEOREM 4. Let $\phi(t)$ be the characteristic function of an infinitely divisble law, and assume that $\phi(t)$ is an analytic function. Then $\phi(t)$ has no zeros inside its strip of convergence.

If $\phi(z)$ is the characteristic function of an infinitely divisible law, then the function $[\phi(z)]^{1 / n}$ must be a characteristic function for any $n$, and also a factor of $\phi(z)$. If furthermore $\phi(z)$ is also assumed to be analytic, then, by the Lévy-Raikov theorem, $[\phi(z)]^{1 / n}$ must be analytic at least in the strip of convergence of $\phi(z)$. If $\phi(z)$ were to have a zero at some point $z_{0}$, then $[\phi(z)]^{1 / n}$ 
would have a singularity at $z_{0}$ for sufficiently large $n$, which is impossible.

We can use Theorem 4 in the construction of an example which shows that an infinitely divisible law can be obtained as the product of two noninfinitely divisible laws. Let

$$
\phi(t)=\left[\left(1+\frac{i t}{v}\right)\left(1+\frac{i t}{\bar{v}}\right)\right] /\left[\left(1-\frac{i t}{a}\right)\left(1-\frac{i t}{v}\right)\left(1-\frac{i t}{\bar{v}}\right)\right], \quad v=a+i b .
$$

A simple computation shows that $\phi(t)$ is a characteristic function if

$$
b \geq 2 \sqrt{2} a
$$

Then also $\phi(-t)$ is a characteristic function, as is

$$
\psi(t)=\phi(t) \phi(-t)=\frac{1}{1+\frac{t^{2}}{a^{2}}}
$$

The characteristic functions $\phi(t)$ and $\phi(-t)$ are analytic characteristic functions with zeros in their strip of convergence: hence they are not characteristic functions of infinitely divisible laws. Their product $\psi(t)$ is the characteristic function of the Laplace distribution, which is known to be infinitely divisible.

\section{REFERENCES}

1. R. P. Boas, Jr., Sur les series et intégrales de Fourier à coefficients positifs, C. R. Acad. Sci. Paris 228 (1949), 1837-1838.

2. H. Cramér, Mathematical methods of statistics, Princeton University Press, 1946.

3. D. Dugué, Analyticité et convexité des fonctions caractéristiques, Ann. Inst. H. Poincaré 12 ( 1951 ), 45-56.

4. R. Fortet, Calcul des moments d'une fonction de répartition à partir de sa caractéristique, Bull. Sci. Math. (2) 68 (1944), 117-131.

5. M. Fréchet, Recherches théoriques modernes sur le calcul des probabilités I, Gauthiers Villars, Paris, 1950.

6. P. Lévy, L'arithmétique des lois de probabilité, C. R. Acad. Sci. Paris 204 (1937), 80-82. 
7. $17-40$.

L'arithmétique des lois de probabilité, J. Math. Pures Appl. 103 (1938),

8. D. A. Raikov, On the decomposition of Gauss and Poisson laws, Izvestiya Akad. Nauk SSSR. Ser. Mat. 1938 (1938), 91 - 124. (Russian. English summary)

[I. А. Райков, о разлоиении законое Іаусса и Пуассона, Иввестия Академии Наук СССР, сер. матем. 1938 (1938), 91-124.]

9. D. V. Widder, The Laplace transform, Princeton University Press, 1946.

National Bureau of Standards, Washington

University of Cincinnati 
